\title{
Lessons for life
}

\author{
Elizabeth Loder head of research
}

The BMJ

As children around the world trudge back to school, it's worth considering the connection between education and health. Better educated people have a lower risk of myocardial infarction, stroke, and even certain kinds of cancer. Is education the cause of these better outcomes, or is it just a marker of people who are destined to be healthier for other reasons?

Tillmann and colleagues (doi:10.1136/bmj.j3542) used mendelian randomisation to see whether people with genetic variants associated with longer education have a lower risk of coronary heart disease. And they do: 3.6 years' extra education was associated with a roughly one third lower risk. The researchers say that these findings "suggest that low education is a cause of coronary heart disease."

Editorialists Richards and Evans (doi:10.1136/bmj.j3849) find this evidence convincing. They remind us how the technique of mendelian randomisation works: genes linked to longer time in education are distributed randomly at birth. Analysing people on the basis of their genetic make-up helps separate the health effects of education from those of affluence, intelligence, or the many other advantages that track closely with years of schooling. In this study, the benefit conferred by extra education was "comparable to the effect of short term statin use for primary prevention of coronary heart disease." Furthermore, the results are in line with other research including natural experiments. The evidence for compulsory education as a public health intervention is growing stronger.
Meanwhile, one lesson we can't seem to learn is that more medical technology isn't always a good thing. Quinonez and colleagues (doi:10.1136/bmj.j3850) review the increased use of pulse oximetry to assess children with bronchiolitis. Oximetry is associated with a large increase in hospital admissions but no improvement in mortality. The authors suggest that overdiagnosis of hypoxaemia could be reduced by using this technology more judiciously, lowering the oxygen level that triggers medical intervention, and intermittent instead of continuous monitoring in some settings.

Jeremy Hunt is a politician, not a scientist, but that did not keep him from picking a fight with Stephen Hawking about hospital staffing. Some remedial lessons about cherry picking evidence may be in order for Hunt: Rachel Clarke (doi:10.1136/bmj. j3972) views his Twitter spat with Hawking as "deadly serious" rather than silly. Selective use of evidence in the service of ideology undermines science, she says, and, "in an era of fake news ... facts are all we have."

Finally, Margaret McCartney (doi:10.1136/bmj.j3955) declares that "General practice is based on a lie-a lie that we can do this safely and well in 10 minutes." Double that time would be needed for "acceptably safe" practice; even more if patients are to be fully involved in their care.

"No one goes into medicine for an easy life. But I'm haunted by a feeling of persistent failure," writes McCartney. Doctors know that no amount of education or skill can make up for a lack of time or resources. It's a lesson policy makers would do well to learn, too. 